\title{
NOTES
}

\section{Rickettsia massiliae sp. nov., a New Spotted Fever Group Rickettsia}

\author{
L. BEATI AND D. RAOULT* \\ Unité des Rickettsies, Faculté de Médecine, 27 Boulevard Jean Moulin, \\ 13385 Marseille Cedex 5, France
}

\begin{abstract}
We propose the name Rickettsia massiliae sp. nov. (type strain, Mtu1 in the Collection of the World Health Organization Collaborative Center for Rickettsial Reference, Marseille, France) for a spotted fever group rickettsia determined to be distinct from previously recognized species by the serotyping method $(L$. Beati, J.-P. Finidori, B. Gilot, and D. Raoult, J. Clin. Microbiol. 30:1922-1930, 1992). This rickettsia has biological characteristics similar to those of the other spotted fever group rickettsiae. In addition, a sodium dodecyl sulfate-polyacrylamide gel electrophoresis protein analysis, a polymerase chain reaction followed by a restriction fragment length polymorphism analysis of DNA fragments, and pulsed-field electrophoresis of the genome of $\boldsymbol{R}$. massiliae revealed unique migration patterns distinct from those of all previously recognized spotted fever group rickettsiae. These additional characteristics (Beati et al., J. Clin. Microbiol. 30:1922-1930, 1992), together with the data usually considered sufficient for description of rickettsiae, are crucial to the proposal of this new species and should be helpful in species identification.
\end{abstract}

In this paper, we formally name a rickettsia isolated from the hemolymph of ticks (3). The first isolate, strain Mtu1 ${ }^{\mathrm{T}}(\mathrm{T}$ = type strain) was isolated in 1990 from a Rhipicephalus turanicus tick in an area near Marseille, France. Since then, another strain has been isolated from a Rhipicephalus sanguineus tick (brown dog tick) collected in Sisteron, France, and other strains have been detected in a Rhipicephalus sanguineus tick from Portugal (1) and in Rhipicephalus mushamae, Rhipicephalus sulcatus, and Rhipicephalus lunulatus ticks collected in the Central African Republic (2).

As determined by the serotyping method (5), we found that the new isolates were distinct from all previously described spotted fever group (SFG) rickettsiae (15 species). Cross-titrations of murine polyclonal sera with these new strains and with the other SFG rickettsiae $(3,4)$ revealed 6 to 13 specific differences between the new organisms and the other SFG rickettsiae $(3,4)$.

The results of a sodium dodecyl sulfate-polyacrylamide gel electrophoresis (SDS-PAGE) protein analysis revealed different electrophoretic mobilities for the major immunodominant high-molecular-weight surface polypeptides of strain $\mathrm{Mtu}^{\mathrm{T}}$ compared with the antigens of the standard SFG rickettsiae, including Rickettsia slovaca 13-B, Rickettsia sibirica 232, Rickettsia conorii Moroccan, Rickettsia rickettsii Sheila Smith, Rickettsia rhipicephali 2-7-6, Rickettsia montana ATCC VR-611, Rickettsia helvetica $\mathrm{C}^{\mathrm{T}}{ }^{\mathrm{T}}$, Rickettsia parkeri Maculatum 20, Israeli spotted fever rickettsia strain ISTT CDC-1, Rickettsia japonica YM, Thai tick typhus rickettsia strain TT-118, Rickettsia akari MK-Kaplan, and Rickettsia australis Phillips $(3,4)$.

Genetic analyses of the citrate synthase and 190-kDa protein genes (7) of strain $\mathrm{Mtu}^{\mathrm{T}}$ amplified by the polymerase chain reaction and digested with restriction endonucleases (restriction fragment length polymorphisms) revealed profiles different from those of other SFG rickettsiae

${ }^{*}$ Corresponding author.
$(3,4,7)$. When the citrate synthase gene amplified material was digested by $A l u \mathrm{I}$, it produced a unique profile. The migration patterns for the genome of strain $\mathrm{Mtu1}^{\mathrm{T}}$ digested with different enzymes (SmaI, EagI, and BssHII) and analyzed by pulsed-field electrophoresis were clearly different from the profiles obtained for the reference SFG rickettsiae (8).

Therefore, strain $\mathrm{Mtu}^{\mathrm{T}}$ can be considered a new species distinct from all previously recognized SFG rickettsiae, not only on the basis of the usual serological criteria, but also on the basis of the results of two different genetic analyses $(3,4$, 8). The polymerase chain reaction-restriction fragment length polymorphism profile of $\mathrm{Mtu1}^{\mathbf{T}}$, obtained after amplification of the citrate synthase gene and digestion with AluI, is unique and distinct from the profile found for all previously described SFG rickettsiae. Therefore, we presume that the difference between this strain and the classically defined SFG rickettsiae is more important than the difference between strains in the SFG $(3,7)$.

Furthermore, from an epidemiological point of view, the geographical distribution of this isolate seems to be very wide, ranging from the Mediterranean area and Portugal to Central Africa (1-3).

On the basis of the distinctive properties described above we propose that the new strains should be named Rickettsia massiliae.

Description of Rickettsia massiliae sp. nov. Rickettsia massiliae (mas.si' li.ae. L. gen. n. massiliae, from Massilia, the Latin name of Marseille, France, where the organism was first isolated) is an obligately gram-negative intracellular organism. It grows in tissue cultures of L929, Vero, or human embryonic lung fibroblast cells. Gimenez-stained infected cells contain small, intracellular, rod-shaped or diplobacillary bacteria that are slightly shorter $(0.3$ to 0.4 by 0.6 to $1 \mu \mathrm{m}$ ) than the other SFG rickettsiae (3). This organism can also be detected in infected cells by indirect immunofluorescence by using human immune sera reactive with the lipopolysaccharide of Rickettsia conorii, a cross- 
reaction which has previously been demonstrated by a Western blot (immunoblot) analysis $(3,6)$.

This rickettsia possesses an outer slime layer and a trilaminar cell wall, as determined by electron microscopy. It grows free in the cytoplasm of the cells without membrane enclosure and is not present in nuclei (3).

Pathogenicity for humans or animals has not been demonstrated. Male Hartley's guinea pigs show no symptoms after inoculation of $10^{5}$ organisms.

The results of microimmunofluorescence serotyping and SDS-PAGE protein analysis of purified organisms, as well as polymerase chain reaction-restriction fragment length polymorphism and pulsed-field electrophoresis genetic analyses strain $\mathrm{Mtu}^{\mathbf{T}}$, show that this rickettsia is different from all previously described SFG rickettsiae.

Type strain. Type strain Mtul has been deposited in the Collection of the World Health Organization Collaborative Center for Rickettsial Reference, Marseille, France.

This work was supported by the Fonds National Suisse de la Recherche Scientifique, by the Société Académique Vaudoise, and by the Région PACA.

\section{REFERENCES}

1. Bacellar, F. (Instituto Nacional de Saúde, Dr Ricardo Jorge, Centro de Estudos de Zoonoses, Aguas de Moura, Portugal). 1992. Personal communication.
2. Beati, L., M. Eremeeva, X. J. Yu, H. Tissot Dupont, and D. Raoult. 1992. Rickettsia massiliae (sp. nov.). Abstr. 10th SesquiAnnu. Meet. Am. Soc. Rickettsiol. Rickettsial Dis.

3. Beati, L., J.-P. Finidori, B. Gilot, and D. Raoult. 1992. Comparison of microimmunofluorescence serologic typing, sodium dodecyl sulfate-polyacrylamide gel electrophoresis, and polymerase chain reaction followed by restriction fragment length polymorphism analysis for identification of rickettsiae: characterization of two new rickettsial strains. J. Clin. Microbiol. 30:1922-1930.

4. Beati, L., O. Péter, W. Burgdorfer, A. Aeschlimann, and D. Raoult. 1993. Confirmation that Rickettsia helvetica sp. nov. is a distinct species of the spotted fever group of rickettsiae. Int. J. Syst. Bacteriol. 43:521-526.

5. Philip, R. N., E. A. Casper, W. Burgdorfer, R. K. Gerloff, L. E. Hughes, and E. J. Bell. 1978. Serologic typing of rickettsiae of the spotted fever group by indirect microimmunofluorescence. J. Immunol. 121:1961-1968.

6. Raoult, D., and G. A. Dasch. 1989. Line blot and Western blot immunoassays for diagnosis of Mediterranean spotted fever. J. Clin. Microbiol. 27:2073-2079.

7. Regnery, R. L., C. L. Spruill, and B. D. Plikaytis. 1991. Genotypic identification of rickettsiae and estimation of intraspecies sequence divergence for portions of two rickettsial genes. J. Bacteriol. 173:1576-1589.

8. Roux, V., and D. Raoult. Genotypic identification and phylogenetic analysis of the spotted fever group rickettsiae using pulsedfield gel electrophoresis. J. Bacteriol., in press. 